\title{
Resultados en el corto y mediano plazo del tratamiento endovascular en la enfermedad arterial obstructiva infrapoplítea en pacientes con isquemia crítica de una extremidad
}

\author{
Daniel García O. ${ }^{1,2}$, Juan Bombin F. ${ }^{1,2}$, Angela Poblete S. ${ }^{1,2}$, \\ Alfonsina Concha A. ${ }^{3}$ e Iver Zegarra I. ${ }^{1}$
}

\section{Short to midterm results after endovascular approach in critical limb isquemia in patients with below-the-knee arterial disease}

\begin{abstract}
Aim: Show initial and midterms results of endovascular Percutaneous Transluminal Angioplasty (PTA) in critical limb isquemia (CLI) patients caused by below-the-knee arterial disease. Materials and Method: Observational, descriptive and retrospective study. 42 CLI patients admitted in our hospital from 2009 until 2018 with Fontaine III or IV treated by PTA in infrapopliteal arteries were analyzed, collecting demographic, clinical and surgical characteristics, additional procedures in Fontaine IV, hospital stay, postoperative complications, need of reintervention, limb preservation and mortality with one year follow-up after procedure. Results: 42 patients, average age 66 year-old (46-82), with significant comorbidities. Fontaine IV stage patients were $83.3 \%$. In 16 cases more than one artery was intervened. No stent revascularization was performed. Complications occurred in 3 patients, 2 required major amputation and an urgent endovascular reintervention was required in another. Average hospital stay was 22 days with no post-operative mortality. One-year global mortality was 9.5 One-year follow-up in 35 patients shows that $77 \%$ preserved their limb. Conclusion: Percutaneous transluminal angioplasty procedure in this patients has a high rate of limb preservation in a one-year follow-up. There was no post-operative mortality.

Key words: critical limb ischemia; infrapopliteal; angioplasty.
\end{abstract}

\section{Resumen}

Objetivo: Mostrar los resultados en el corto y mediano plazo del tratamiento endovascular de angioplastia transluminal percutánea (ATP) con balón en pacientes en estado de isquemia crítica por enfermedad arterial obstructiva infrapoplítea. Materiales y Método: Estudio descriptivo, observacional, retrospectivo. Se incluyeron los pacientes hospitalizados entre 2009 y 2018 por isquemia crítica Fontaine III o IV sometidos a una ATP del territorio infrapoplíteo. Se observó como objetivos primarios la preservación de la extremidad afectada y la mortalidad posoperatoria a un año plazo, y como objetivos secundarios los procedimientos adicionales en pacientes con lesiones o necrosis distales, estadía hospitalaria, complicaciones posoperatorias y necesidad de reintervención. Resultados: Se incluyeron 42 pacientes con un promedio de edad de 66 años (46-82), con importantes comorbilidades. Un 83,3\% ingresó en etapa Fontaine IV. En 16 casos se realizó una angioplastia percutánea en más de una arteria. No se colocó stents. Se presentaron complicaciones en 3 pacientes, 2 requirieron una amputación mayor y en otro se debió efectuar un nuevo procedimiento endovascular de rescate. La estadía hospitalaria promedio fue 22 días. No hubo mortalidad precoz posprocedimiento. La mortalidad global a un año fue 9,5\%. A todos los pacientes en etapa Fontaine IV se les efectuó algún procedimiento adicional, a 31 una amputación menor, 3 cerraron sus lesiones por segunda intención y en otro se realizó un injerto dermo-epidérmico. De los 35 pacientes con seguimiento, $77 \%$ preservó su extremidad a un año. Conclusión: La reparación endovascular mediante una angioplastia percutánea en estos casos es un procedimiento seguro y tiene una alta tasa de preservación de la extremidad inicial a un año de seguimiento.

Palabras clave: isquemia crítica; infrapoplítea; angioplastia percutánea.
'Servicio de Cirugía, Hospital Dr. Eduardo Pereira. Valparaíso, Chile. Departamento de Cirugía, Universidad de Valparaíso. Valparaíso, Chile. ${ }^{3}$ Interna de Medicina, Universidad de Valparaíso. Valparaíso, Chile.

Recibido 2020-06-01 y aceptado 2020-09-20

Correspondencia a: Dr. Daniel García O. d.garciaoneto@gmail.com 


\section{Introducción}

La isquemia crítica de extremidades inferiores afecta cada vez a más pacientes. Una población con mayor esperanza de vida, el creciente número de diabéticos, pacientes con enfermedad renal crónica y fumadores, son factores que contribuyen a este grado tan severo de la enfermedad vascular periférica ${ }^{1}$. La incidencia de la isquemia crítica es entre 500 a 1.000 nuevos casos por cada millón de habitantes todos los años en Europa y Estados Unidos ${ }^{2}$.

La isquemia crítica se manifiesta por dolor permanente de reposo en el pie o pierna afectada, úlceras cutáneas, que no cicatrizan en un plazo mayor a dos semanas, o necrosis de ortejos, condición que pone en riesgo la viabilidad de la extremidad debido al aporte sanguíneo insuficiente. Según la clasificación de Fontaine de la isquemia crónica de las extremidades inferiores, existen IV estadios, de los cuales el III (dolor de reposo) y el IV (úlceras o necrosis) representan la isquemia crítica con amenaza de pérdida de la extremidad ${ }^{3}$.

La base anatomopatológica es la ateromatosis, la cual genera estenosis y finalmente oclusión de las arterias de las extremidades inferiores. La severidad clínica de esta enfermedad dependerá de la extensión y el grado de estenosis arterial, la presencia de circulación colateral y el número de arterias comprometidas. El manejo de la isquemia crítica tiene como objetivo principal evitar la pérdida de la extremidad. Es tanto así que el porcentaje de amputaciones mayores en pacientes con isquemia crítica varía entre el $14,5 \%$ al $46,4 \%$.

En la mayoría de los pacientes, sobre todo en aquellos con diabetes mellitus y enfermedad renal crónica, la enfermedad arterial periférica es multinivel, incluyendo compromiso de arterias de mediano y pequeño calibre de la pierna y el pie. La enfermedad infrapoplítea se presenta en el $60 \%$ al $80 \%$ de los pacientes con isquemia crítica. Las lesiones infrapoplíteas significativas disminuyen en forma importante la posibilidad de revascularización ${ }^{6}$, aumentando el riesgo de pérdida de la extremidad.

Es por esto por lo que el abordaje mínimamente invasivo endovascular ha ganado interés en los últimos 10 años ${ }^{7}$, pues es capaz de manejar vasos de pequeño calibre con altas tasas de éxito, pocas complicaciones y mortalidad asociada, entre ellas, la angioplastía transluminal percutánea (ATP) con balón, que permite la dilatación de un vaso estenótico, con o sin el uso adicional de un stent ${ }^{8}$.

El objetivo de este trabajo es mostrar los resultados inmediatos y a un año de seguimiento en cuanto a mortalidad y preservación de la extremidad comprometida, y otros parámetros, en pacientes que presentaban una isquemia crítica por enfermedad arterial obstructiva infrapoplítea tratados de forma endovascular con ATP desde enero de 2009 a diciembre de 2018 por el equipo de cirugía vascular de nuestro hospital. No encontramos estudios en Chile que hayan evaluado los resultados de esta técnica a nivel infrapoplíteo.

\section{Materiales y Método}

Presentamos un estudio descriptivo, observacional, retrospectivo de 42 pacientes consecutivos del Servicio de Cirugía del Hospital Dr. Eduardo Pereira de Valparaíso ingresados por isquemia crítica de una extremidad inferior (Fontaine III o IV) y que fueron sometidos a una angioplastia transluminal percutánea en el territorio infrapoplíteo en forma exclusiva, entre enero de 2009 y diciembre de 2018. Se excluyeron los pacientes que presentaron algún otro procedimiento en el nivel aortoilíaco y femoropoplíteo.

Consideramos para efectuar una angioplastia transluminal percutánea a pacientes portadores de enfermedad arterial periférica en una extremidad que presentaron dolor de reposo permanente y especialmente nocturno (etapa III de Fontaine) y aquellos con lesiones ulceradas o necrosis de ortejos o segmentos distales de la extremidad (etapa IV de Fontaine), todos con clara amenaza de pérdida de su extremidad al momento de la consulta.

Se les efectuó pesquisa clínica y de laboratorio de comorbilidades asociadas, y se les realizó un estudio angiográfico. Todos los casos con enfermedad arterial obstructiva distal en el sector infrapoplíteo fueron incluidos en esta casuística. Se excluyeron los pacientes que requerían algún procedimiento en el nivel aortoilíaco y femoropoplíteo. Los pacientes firmaron un consentimiento informado y aceptaron tener un control médico periódico y permanente.

El procedimiento endovascular se realizó en un pabellón híbrido con las debidas condiciones de esterilidad, protección radiológica y monitorización. Se usó anestesia local en la zona de acceso percutáneo de la arteria femoral común en la región inguinal. En todos los pacientes se inició una doble antiagregación plaquetaria con ácido acetilsalicílico y clopidogrel previo al procedimiento. La punción femoral fue hecha con visión ecográfica, en el mismo lado de la lesión en forma anterógrada, o en el lado contralateral en forma retrógrada cruzando las guías y elementos hacia el lado comprometido a 
través de las arterias ilíacas y aorta distal. Inmediatamente logrado el acceso arterial se indicó heparina no fraccionada en dosis de 100 unidades/kilogramo de peso. Para llegar hasta las arterias de la pierna se usó guías $0,035^{\prime}$ en el muslo y 0,014' en la pierna. En algunos pocos casos fue necesaria una punción retrógrada muy distal en una arteria tibial posterior o pedia para pasar una guía 0,014 ' traspasando la obstrucción arterial, y recogiéndola con un lazo llevado desde la zona femoral para efectuar después la angioplastia. Las guías se avanzaron bajo visión angiográfica usando pequeñas dosis de medio de contraste en cada segmento. Una vez que la guía traspasó la lesión obstructiva se efectuó la dilatación con balones no complacientes de diversos diámetros de acuerdo con el tamaño de la arteria. Siempre se realizó una angiografía de control final. No revertimos el efecto de la heparina. La hemostasia del sitio de punción se realizó por compresión local, aunque se usó ocasionalmente un dispositivo de cierre percutáneo. Los pacientes quedaron con un apósito compresivo y en reposo absoluto por $12 \mathrm{~h}$.

A los pacientes con lesiones ulceradas o necrosis, se les realizó amputaciones menores o injertos de piel bajo anestesia local o general, o se esperó el cierre espontáneo de las heridas con curaciones.

Posteriormente todos los pacientes fueron sometidos a controles posoperatorios clínicos inmediatos, al día siguiente y posteriormente al mes, 6 meses y anual. Se mantuvo la doble antiagregación plaquetaria por seis meses y luego se continuó con solo un antiagregante, habitualmente ácido acetilsalicílico. No se indicaron anticoagulantes.

Los datos fueron recolectados a través de revisión de fichas clínicas y, posteriormente, se recopilaron en una base de datos en el software Microsoft Excel $^{\circledR}$ 2011. Se realizó análisis estadístico descriptivo a través de tabulación de datos, cálculo de medidas de tendencia central y de frecuencia relativa con el paquete estadístico Stata 13. Durante la recolección de datos el principio de beneficencia y no maleficencia fueron los ejes centrales, extrayendo información conservando el anonimato de los participantes y sin la necesidad de procedimientos adicionales para la evaluación de resultados.

Los siguientes datos fueron recolectados:

1. Edad, sexo y comorbilidades (diabetes mellitus, hipertensión arterial, enfermedad renal crónica y tabaquismo).

2. Grado de isquemia crónica según la clasificación de Fontaine.

3. Arteria revascularizada.

4. Necesidad de revascularización adicional, ya sea endovascular o abierta.
5. Procedimiento adicional en pacientes con isquemia crítica Fontaine IV.

6. Complicaciones perioperatorias.

7. Estadía hospitalaria.

8. Seguimiento a un año desde el procedimiento:

a. Preservación de la extremidad.

b. Amputación.

9. Mortalidad a un año desde el procedimiento.

\section{Resultados}

Se obtuvo una muestra total de 42 pacientes (31 hombres) con promedio de edad de 66 años (rango 46-82 años). Gran parte de los pacientes eran portadores de alguna patología asociada, destacando la hipertensión arterial en 40, diabetes mellitus en 36, tabaquismo en 24 y enfermedad renal crónica avanzada en 12. La mayoría, 35 pacientes correspondieron a Fontaine IV, es decir, con pérdida de tejido o necrosis, mientras que sólo 7 fueron Fontaine III, es decir, presentaban sólo dolor de reposo.

Sólo se efectuaron angioplastias percutáneas con balones simples, sin drogas, tampoco se colocaron stents. En 16 pacientes se efectuó una angioplastia en más de una arteria. Se trataron 58 arterias en los 42 pacientes. Del total de arterias dilatadas, el $36,2 \%$ (21) correspondió al tronco tibioperoneo, el $25,8 \%$ (15) a la arteria tibial anterior, el 22,4\% (13) a la arteria tibial posterior y el 15,6\% (9) a la arteria peronea (Tabla 3 ).

Tabla 1. Descripción general de la edad y factores de riesgo

\begin{tabular}{|lcc|}
\hline Pacientes & 42 & \\
Edad promedio & 66 años & $46-82$ años \\
Factores de riesgo $(\mathrm{N}=42)$ & $\mathbf{n}$ & $\mathbf{\%}$ \\
$\quad$ Sexo masculino & 31 & 74 \\
Diabetes mellitus & 36 & 85,7 \\
Hipertensión arterial & 40 & 95,2 \\
Enfermedad renal crónica & 12 & 28,5 \\
Tabaquismo & 24 & 57,1 \\
\hline
\end{tabular}

Tabla 2. Clasificación de Fontaine

\begin{tabular}{|lcc|}
\hline Fontaine $(\mathbf{N}=\mathbf{4 2})$ & $\mathbf{n}$ & $\mathbf{\%}$ \\
\hline III & 7 & 16,7 \\
IV & 35 & 83,3 \\
\hline
\end{tabular}




\section{ARTÍCULO ORIGINAL}

Tabla 3. Arteria comprometida

\begin{tabular}{|lcc|}
\hline Arteria comprometida $(\mathbf{N}=\mathbf{5 8})$ & $\mathbf{n}$ & $\mathbf{\%}$ \\
\hline Tronco tibioperoneo & 21 & 36,2 \\
Arteria tibial anterior & 15 & 25,8 \\
Arteria tibial posterior & 13 & 22,4 \\
Arteria peronea & 9 & 15,6 \\
\hline
\end{tabular}

Tabla 4. Resultados con seguimiento a 1 año

\begin{tabular}{|lcc|}
\hline Variable & n & \% \\
Mortalidad $(\mathrm{N}=42)$ & 4 & 9,5 \\
Preservación de la extremidad $(\mathrm{N}=37)$ & & \\
$\quad$ Sí & 27 & 77 \\
No & 10 & 33 \\
Tratamiento adicional Fontaine IV $(\mathrm{N}=35)$ & & \\
Amputación de 1 ortejo & 17 & 48,6 \\
Amputación de 2 ortejos & 8 & 22,9 \\
Amputación transmetatarsiana & 6 & 17,1 \\
Cierre por segunda intención & 3 & 8,5 \\
Injerto dermo-epidérmico & 1 & 2,9 \\
\hline
\end{tabular}

En todos los pacientes se logró un acceso arterial satisfactorio. No hubo hematomas, sangrados o pseudoaneurismas en el sitio de punción arterial, sólo equimosis transitorias. Se logró realizar la angioplastia percutánea con balón en todos los pacientes. Tres pacientes presentaron alguna complicación relacionada al procedimiento, de ellos, 2 requirieron una amputación mayor durante su estadía hospitalaria por gangrena húmeda secundaria a una infección "ascendente" de una lesión infectada del pie (una supracondílea y una infracondílea). Otro paciente presentó disección arterial posangioplastía, que se corrigió con un nuevo procedimiento endovascular con resultado satisfactorio.

En los pacientes con isquemia crítica Fontaine IV, a 17 pacientes se realizó amputación de un ortejo, en 8 se realizó amputación de 2 ortejos, en 6 pacientes se le efectuó una amputación transmetatarsiana, en 3 se logró un cierre de las heridas por segunda intención y en un caso se realizó un injerto dermo-epidérmico de espesor parcial. Todos con buena evolución, cicatrizaron sus heridas, los pacientes volvieron a caminar y no tuvieron necesidad de otra intervención (Tabla 4).
La estadía hospitalaria promedio fue de 22 días con un máximo de 121 días y un mínimo de 1 día (Tabla 4).

No hubo mortalidad a los 30 días del procedimiento.

Se logró el seguimiento hasta un año en 35 pacientes. No se logró el seguimiento en 7 pacientes, 4 fallecieron y en 3 no se consiguió contactar al paciente. De los 35 pacientes con seguimiento a un año, en un 77\% (27) se logró la preservación de la extremidad (Tabla 4). La mortalidad global a 1 año de seguimiento fue de $9,5 \%$ (4 pacientes), y las muertes fueron secundarias a causas no relacionadas con la enfermedad arterial periférica.

\section{Discusión}

Se requiere una actitud activa frente a un paciente con isquemia crítica, por el elevado riesgo de pérdida de la extremidad si no se efectúa una pronta revascularización. En un estudio retrospectivo que incluyó 69 pacientes con isquemia crítica sin revascularización, se observó que sólo el 5\% pudo preservar su extremidad en un período de 1 año ${ }^{9}$.

En los últimos años se ha acumulado bastante evidencia que apoya la utilización de estrategias de revascularización endovascular en pacientes con isquemia crítica, con un especial interés en los pacientes con compromiso infrapoplíteo exclusivo. De hecho, muchos grupos consideran esta estrategia como la modalidad primaria de revascularización en pacientes con isquemia crítica teniendo en cuenta la mayor seguridad de la técnica endovascular por ser un procedimiento mínimamente invasivo, en pacientes que presentan una mayor comorbilidad y riesgo perioperatorio. Por otra parte, se puede efectuar igual una cirugía abierta aún en caso de falla de la técnica endovascular. La utilidad de la angioplastia percutánea es otorgar una adecuada perfusión tisular, aunque sea sólo temporal, y así permitir la cicatrización de los tejidos y lograr la preservación de la extremidad. La reestenosis no influye en la pérdida de la extremidad una vez que se ha producido la cicatrización de la herida ${ }^{10,11}$

Gran parte de estos pacientes, además, presentan múltiples enfermedades asociadas como diabetes mellitus, hipertensión arterial, enfermedad renal crónica y tabaquismo, pero también presentan compromiso ateroesclerótico a nivel cerebral y cardiaco, lo que los hace malos candidatos para intervenciones quirúrgicas complejas como los puentes distales por la alta morbimortalidad perioperatoria, la pobre per- 
meabilidad a largo plazo de esas intervenciones y la imposibilidad técnica de realizar un procedimiento clásico de revascularización ${ }^{9,12}$.

Los estudios que han comparado los resultados de la reparación endovascular versus la reparación abierta son pocos. Una revisión del Cochrane Database de resultados de estudios no randomizados no mostró diferencias significativas entre ambas técnicas ${ }^{13}$. El único trabajo chileno que evaluó la angioplastia percutánea en el manejo de la isquemia crítica es el de Hasbún et al., de 2008, en que se incluyeron pacientes a quienes se le realizaron procedimientos en el sector femoropoplíteo en forma exclusiva ${ }^{14}$.

En nuestro estudio la preservación de la extremidad a un año desde el procedimiento se logró en el $77 \%$ de los pacientes, lo cual es similar a lo publicado en la literatura, que va de un $74,2 \%$ a un $96,6 \%$. La mayor preservación de una extremidad del $96,6 \%$ a 1 año fue comunicado por Bosiers et al., en el año $2006^{15,16}$. Esto podría deberse a que un $80,1 \%$ de los pacientes incluidos en su estudio presentaban sólo dolor de reposo (Fontaine III), habiendo pérdida de tejido (Fontaine IV) en 19,9\% de los pacientes. En nuestro estudio, el 83,3\% presentaban pérdida de tejido y solo un $17,7 \%$ dolor de reposo. En nuestra serie los pacientes tenían mayor daño tisular, debido probablemente a una enfermedad más avanzada y una consulta más tardía. Es posible que una derivación más temprana de estos pacientes al cirujano vascular mejoraría estos resultados.

De los pacientes intervenidos, 3 presentaron una complicación relacionada con el procedimiento, lo que representa el 7,1\% del total de pacientes. De ellos, 2 requirieron una amputación mayor, y otro paciente requirió un nuevo procedimiento endovascular. No existieron casos de complicaciones sistémicas ni muerte durante la estadía hospitalaria, lo cual apoya al procedimiento endovascular infrapoplíteo como un tratamiento mínimamente invasivo adecuado, aún en pacientes de alto riesgo general. En la literatura se han reportado complicaciones secundarias al procedimiento mayores al $10 \%{ }^{17}$.

Del total de pacientes, $87,5 \%$ presentaban diabetes mellitus. Asimismo, todos los pacientes que presentaron complicaciones eran diabéticos, similar a lo reportado en la literatura ${ }^{15}$. Diversos estudios han objetivado esta patología como un factor independiente de complicaciones posoperatorias, incluyendo amputación y trombosis 9 .

La mortalidad global a 1 año desde el procedimiento fue del $9,5 \%$ del total de pacientes in- tervenidos, lo cual es similar a lo reportado en la literatura ${ }^{18}$. Las muertes fueron secundarias a causas no relacionadas a la enfermedad arterial periférica. En relación con los pacientes con isquemia crítica Fontaine IV, una vez revascularizados se les realizó diversos procedimientos quirúrgicos logrando la completa cicatrización en todos, quienes pudieron volver a caminar, no siendo necesaria alguna otra intervención.

Dentro de las limitantes del estudio cabe mencionar el reducido tamaño muestral. Además, las características propias del tipo del estudio limitan su validez externa y lo exponen a sesgos intrínsecos a éste, incluyendo selección de la muestra y pérdida de seguimiento de los pacientes. Bajo este argumento los resultados del estudio no podrían ser extrapolables a otras poblaciones, por lo que se requieren más estudios y con mayor nivel de evidencia.

\section{Conclusión}

La reparación endovascular, mediante una angioplastia percutánea en pacientes con isquemia crítica por compromiso arterial obstructivo distal infrapoplíteo es un procedimiento seguro y con un alto éxito técnico.

Se obtuvo una alta tasa de preservación de la extremidad afectada inicialmente a un año de seguimiento, a pesar de haber una alta incidencia de comorbilidades crónicas graves y un estado avanzado de la enfermedad arterial manifestado por la presencia de lesiones tisulares en la mayoría de nuestros pacientes.

\section{Responsabilidades éticas}

Protección de personas y animales. Los autores declaran que para esta investigación no se han realizado experimentos en seres humados ni animales.

Confidencialidad de los datos. Los autores declaran que en este artículo no aparecen datos de pacientes.

Conflictos de interés. No existe ningún interés ni soporte financiero o comercial en este manuscrito.

El autor durante la ejecución del estudio perteneció al Servicio de Cirugía del Hospital Dr. Eduardo Pereira de Valparaíso y al Departamento de Cirugía de la Universidad de Valparaíso. 


\section{Bibliografía}

1. Manojlović V, Popović V, Nikolić D, Milosević D, Pasternak J, Kaćanski M. Analysis of associated diseases in patients with acute critical lower limb ischemia. Med Pregl. 2013;66:41-5. doi: 10.2298/ mpns $1302041 \mathrm{~m}$.

2. Shammas NW. Epidemiology, classification, and modifiable risk factors of peripheral arterial disease. Vasc Health Risk Manag. 2007;3:229-34. doi: 10.2147/ vhrm.2007.3.2.229.

3. Varu VN, Hogg ME, Kibbe MR. Critical limb ischemia. J Vasc Surg. 2010;51:23041. doi: 10.1016/j.jvs.2009.08.073.

4. Bosiers M, Peeters P, D'Archambeau O, Hendriks J, Pilger E, Düber C, et al; AMS INSIGHT-absorbable metal stent implantation for treatment of below-theknee critical limb ischemia: 6-month analysis. Cardiovasc Intervent Radiol. 2009;32:424-35. doi: 10.1007/s00270008-9472-8.

5. Teraa M, Conte MS, Moll FL, Verhaar MC. Critical Limb Ischemia: Current Trends and Future Directions. J Am Heart Assoc. 2016;5:e002938. doi: 10.1161/ JAHA.115.002938.

6. Jaff MR, White CJ, Hiatt WR, Fowkes GR, Dormandy J, Razavi M, et al. An Update on Methods for Revascularization and Expansion of the TASC Lesion Classification to Include Below-the-Knee Arteries: A Supplement to the InterSociety Consensus for the Management of Peripheral Arterial Disease (TASC II). Vasc Med. 2015;20:465-78. doi: 10.1177.

7. Veith FJ, Gupta SK, Wengerter KR, Goldsmith J, Rivers SP, Bakal CW, et al. Changing arteriosclerotic disease patterns and management strategies in lower-limbthreatening ischemia. Ann Surg. 1990;212: 402-12. doi: 10.1097/00000658199010000-00003.
8. Conte MS, Bandyk DF, Clowes AW, Moneta GL, Seely L, Lorenz TJ, et al;. Results of PREVENT III: a multicenter, randomized trial of edifoligide for the prevention of vein graft failure in lower extremity bypass surgery. J Vasc Surg. 2006;43:742-751. doi: 10.1016/j. jvs.2005.12.058.

9. Komai H, Obitsu Y, Shigematsu H. Diabetes and old age could affect longterm patency of paramalleolar distal bypass for peripheral arterial disease in Japanese patients. Circ J. 2011;75:2460-4. doi: 10.1253/circj.cj-11-0156.

10. Tefera G, Hoch J, Turnipseed WD. Limb-salvage angioplasty in vascular surgery practice. J Vasc Surg. 2005;41:988-93. doi: 10.1016/j. jvs.2005.03.018.

11. Feiring AJ, Wesolowski AA, Lade S. Primary stent-supported angioplasty for treatment of below-knee critical limb ischemia and severe claudication: early and one-year outcomes. J Am Coll Cardiol. 2004;44:2307-14. doi: 10.1016/j. jacc.2004.09.037.

12. Saqib NU, Domenick N, Cho JS, Marone L, Leers S, Makaroun MS, et al. Predictors and outcomes of restenosis following tibial artery endovascular interventions for critical limb ischemia. J Vasc Surg. 2013;57:692-9. doi: 10.1016/j. jvs.2012.08.115.

13. Hsu CC, Kwan GN, Singh D, Rophael JA Anthony C, van Driel ML. Angioplasty versus stenting for infrapopliteal arterial lesions in chronic limb-threatening ischaemia. Cochrane Database Syst Rev. 2018;12:CD009195. doi: 10.1002/14651858.CD009195.pub2.

14. Hasbun S, Tapia R, Tepper P, Celedón R. Resultados de las angioplastias del sector femoropoplíteo en isquemia crítica. Rev Chil Cir. 2008;60:145-9. http://dxoi. org/10.4067/S0718-40262008000200011.
15. Bosiers M, Hart JP, Deloose K, Verbist J, Peeters P. Endovascular therapy as the primary approach for limb salvage in patients with critical limb ischemia: experience with 443 infrapopliteal procedures. Vascular 2006;14:63-9. doi: 10.2310/6670.2006.00014.

16. Casella IB, Brochado-Neto FC, Sandri Gde A, Kalaf MJ, Godoy MR, Costa VS, et al. Outcome analysis of infrapopliteal percutaneous transluminal angioplasty and bypass graft surgery with nonreversed saphenous vein for individuals with critical limb ischemia. Vasc Endovascular Surg. 2010;44: 625-32. doi: $10.1177 / 1538574410373663$

17. Giles KA, Pomposelli FB, Spence TL, Hamdan AD, Blattman SB, Panossian H, et al. Infrapopliteal angioplasty for critical limb ischemia: relation of TransAtlantic InterSociety Consensus class to outcome in 176 limbs. J Vasc Surg. 2008;48:12836. doi: 10.1016/j.jvs.2008.02.027.

18. Nasr MK, McCarthy RJ, Hardman J, Chalmers A, Horrocks M. The increasing role of percutaneous transluminal angioplasty in the primary management of critical limb ischaemia. Eur J Vasc Endovasc Surg. 2002;23:398-403. doi: 10.1053/ejvs.2002.1615.

19. Schamp KB, Meerwaldt R, Reijnen MM, Geelkerken RH, Zeebregts CJ. The ongoing battle between infrapopliteal angioplasty and bypass surgery for critical limb ischemia. Ann Vasc Surg. 2012;26:1145-53. doi: 10.1016/j. avsg.2012.02.006.

20. Taylor SM, Kalbaugh CA, Blackhurst DW, Kellicut DC, Langan EM 3rd, Youkey JR. A comparison of percutaneous transluminal angioplasty versus amputation for critical limb ischemia in patients unsuitable for open surgery. J Vasc Surg. 2007;45:304-10. doi: 10.1016/j.jvs.2006.09.038. 